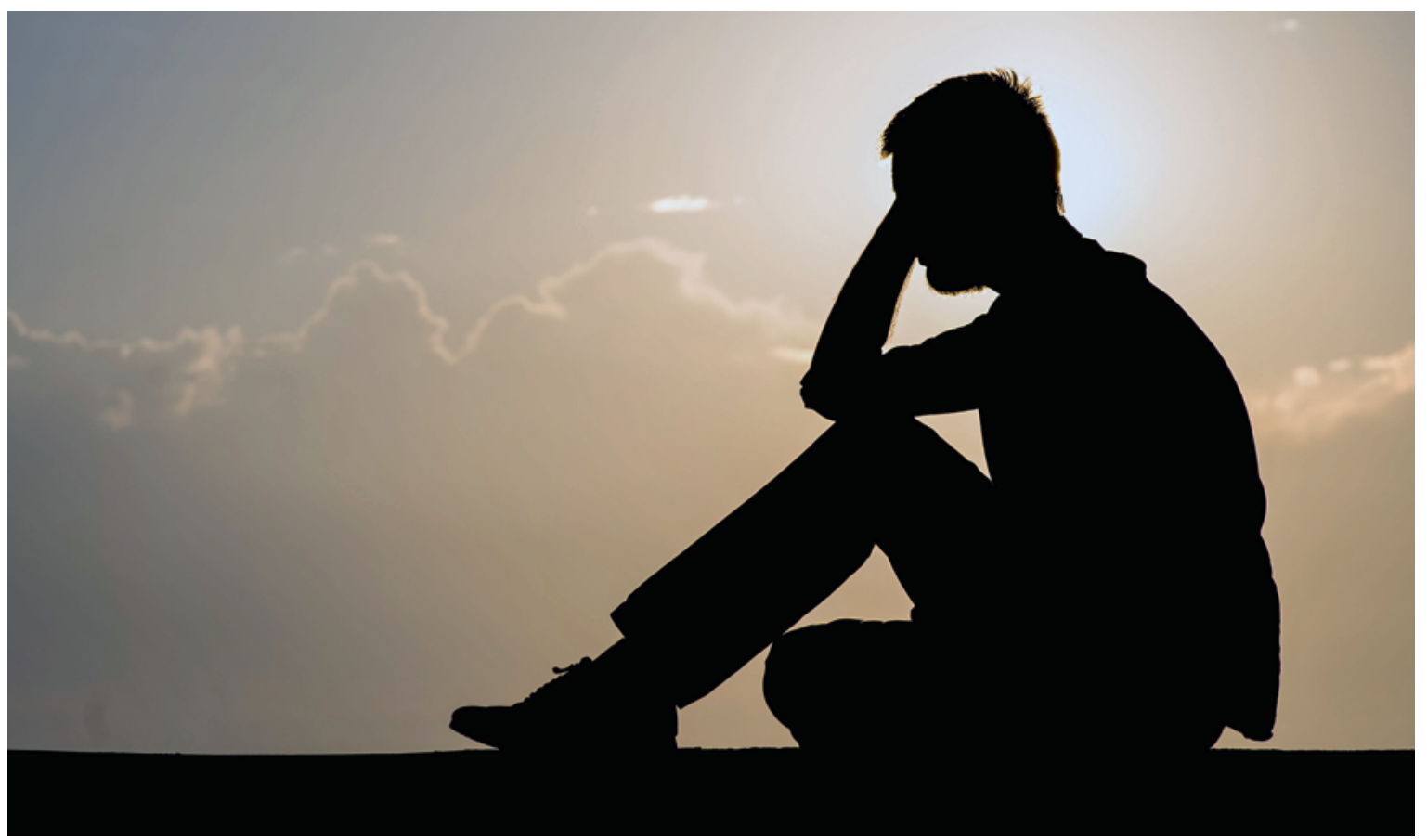

\title{
Nicht jedes Leiden braucht eine Diagnose
}

\section{Rolf H. Adler}

Prof. em. Dr. med., ehem. Chefarzt Innere Medizin, C.-L.-Lory-Haus, Inselspital Bern

Am Donnerstag, den 22. März 2018, erläutern zwei Ärzte im Inselspital anlässlich einer Fortbildung das Chronic Fatigue Syndrome (CFS). Sie bemühen sich, es phänomenologisch zu fassen. Das CFS entzieht sich dieser Bemühung, weil sich weitgehende Überlappungen mit anderen Störungen zeigen, wie der Fibromyalgie, der Myalgischen Encephalomyelitis, dem Chronic Pain Syndrome, dem Burnout, einer Depression, dem Posttraumatic Stress Syndrome, der Multiple Chemical Sensitivity usw.

Keine Studie mit eindeutigen Biomarkern für eines dieser Syndrome kann genannt werden, und keine vernünftige Theorie vermag diese Syndrome zu erklären.

Dazu fällt mir ein, was Mephistopheles im Faust zum wissbegierigen Schüler sagt:

Vor allem haltet Euch an Worte,

dann geht Ihr durch die sich're Pforte, zum Tempel der Gewissheit ein, denn eben wo Begriffe fehlen,

da stellt ein rechtes Wort zur rechten Zeit sich ein.

Richard Feynman, einer der grossen Physiker des 20. Jahrhunderts (1918-1988), meinte zu einer solchen Sachlage: «So schön die Theorie, so intelligent der Forscher, so berühmt sein Name, wenn die Theorie nicht verifiziert werden kann, so soll sie entsorgt werden.» Thomas Henry Huxley (1825-1895), Biologe und Vorkämpfer für Darwins Auffassung zur Entstehung der Arten, äusserte: «The great tragedy of science - is the slaughter of a beautiful hypothesis by an ugly fact.» Also: Es bleibt uns nichts anderes übrig, als die erwähnten «Diagnosen» zu begraben. Was nun? Suchen wir doch Konzepte auf, die uns wohl bekannt sind und die meisten Symptome umfassen, die wir bei den erwähnten Diagnosen beobachten. Es sind das Flucht-KampfMuster von Walter B. Cannon [1], das Rückzug-Konser- 
vierungs-Muster von Schmale und Engel [2], Engels Pain-Proneness [3] und die Konversion [4] bei der Symptombildung. Das Fight-Flight-Konzept umfasst vor allem sympathikotone Prozesse wie Spannung, Schlafstörungen, erhöhten Blutdruck, Tachykardie, Schwitzen kognitive Störungen, Abnahme der Varianz der RR-Abstände im EKG [5] usw. Das Rückzug-KonservierungsKonzept schliesst vor allem vagale Prozesse ein wie Müdigkeit, Histamin-refraktäre Achylie. Beide Muster können auch gemischt auftreten. Hinter der Pain-Proneness und dem chronischen Schmerzsyndrom verbergen sich Kindheitstraumen wie psychischer, physischer und sexueller Missbrauch, Streit zwischen den Eltern, Mutter in der Opferrolle, dem Kind auferlegtes Aggressionsverbot, Zuneigung vor allem wenn das Kind krank oder verletzt ist usw. Die Konversion tritt in belastenden Situationen auf, ist zeitlich mit dem Auftreten der Symptome verbunden, wählt Ort und Art des Symptoms gestützt auf eigene Erfahrungen oder solche bei nahen Bezungspersonen und neutralisiert psychische Konflikte. Als Beispiel einer Konversion sei die 74-jährige Frau erwähnt, die zehn Jahre lang wegen schweren Gesichtsschmerzen erfolglos abgeklärt und behandelt worden ist [6]. Sie leidet an Schmerzen, die im rechten Unterkiefer beginnen, über Nase und linke Stirn bis in die rechte Stirn und die behaarte Kopfhaut ausstrahlen. Das Interview wird mit Einverständnis der Patientin auf Tonband aufgenommen. Nach 19 Minuten komme ich auf den Tod des eingangs des Gesprächs erwähnten, tödlich verunglückten Sohns zu sprechen. Jetzt bricht die Frau in Tränen aus, ruft aus «da hat alles begonnen», schildert, wie ihre Beschwerden vor zehn Jahren drei Wochen nach dem tödlichen Unfall des Sohns begonnen hätten. Sie habe den Verunglückten nach seinem tödlichen Unfall nicht mehr sehen dürfen. Sein Bruder begründete dies mit den Entstellungen im Gesicht des Verunfallten. Bei der Schilderung fährt die Frau mit der rechten Hand genau über die Stellen in ihrem Gesicht, die vom anderen Sohn als verletzt beschrieben worden sind. Ihre Kindheit ist gezeichnet durch Vaters Schläge, Bettnässen und darin, zum Schutz der Mutter zwischen die streitenden Eltern zu stehen. Nach mehreren Sitzungen mit Gesprächen über Trauern wird sie beschwerdefrei. Die heutige Medizin ist charakterisiert durch das Bestreben, eine mehr mathematische Evidenz für die Krankheiten zu finden, daraus Algorithmen abzuleiten, welche die ärztliche Handlung bestimmen sollen. In gewissen Situationen, beispielsweise bei lebensbedrohlichen Zuständen, sind solche Algorithmen sehr nützlich. Es muss zudem die individuelle geschichtliche Evidenz berücksichtigt werden. Wir nehmen deshalb am besten die Bemerkung Søren Kierkegaards als Ausgangspunkt: Wer sein Gegenüber belehren will, wer meint, es sofort zu verstehen, wer sich nicht bescheiden und unvoreingenommen in die Beziehung hineingibt, der ist arrogant, hochnäsig, unbescheiden und besserwisserisch. Praktisch heisst das, dass wir es wagen müssen, dem Patienten ohne Vorwissen über ihn entgegenzutreten, dass wir ihn sprechen lassen, dass wir ihm keine suggestiven Fragen stellen, und dass wir während des Gesprächs auf seine Mimik und Gestik sowie die in uns selbst entstehenden Gefühle achten.

Dieser Haltung begegnen wir bei so verschiedenen Persönlichkeiten wie dem Mathematiker Charles Sanders Peirce (1839-1914), Sigmund Freud (1856-1939) und Conan Doyles Sherlock Holmes. Diese drei empfahlen in unklaren Situationen, sich zurückzulehnen, Gedanken und Gefühle frei flotieren zu lassen und die eigenen Einfälle unzensuriert entgegenzunehmen. Alle drei betonen, wie dieser Zustand es ermöglicht, Lösungen zu finden, die mit den Bemühungen des Verstandes und Intellekts nicht zu finden waren. Das gilt also für die Mathematik, die Psychologie und die Kriminalistik [7].

\section{Schlussfolgerungen}

Lösen wir uns von den Begriffen CFS, Chronic Pain, Burnout usw. Wenden wir uns der individuellen Geschichte des Patienten unvoreingenommen zu, verschonen wir ihn mit diagnostischen Begriffen, an die er sich sonst gern klammert, sie nicht mehr loslässt und weder gefühls- noch verstandesmässig zu seiner eigenen Geschichte findet. Das Gleiche gilt für den Arzt: Verhaftet er bei diesen diagnostischen Begriffen, verbaut er sich den Weg zum Verstehen des Patienten, sucht verbissen durch weitere Abklärungen den kausalen Faktor, findet ihn nicht. Dies führt zu weiteren Abklärungen und Überweisungen an Spezialärzte, die lediglich Zeitverlust und Kosten mit sich bringen.

\section{Bildnachweis}

(C) Esin Deniz | Dreamstime.com

\section{Literatur}

1 Cannon WB. Wut, Hunger, Angst und Schmerz. Eine Physiologie der Emotionen. Urban und Schwarzenberg, München, Berlin, Wien 1975. Originalausgabe: Bodily changes in pain, hunger, fear and rage. D. Appleton and Co., New York and London. 1915

2 Engel GL, Schmale AH. A psychoanalytic theory of somatic disorders. J Am Psychoanal Assoc. 1969;15:344-65.

3 Engel GL. Psychogenic pain and the pain-prone patient. Am J Med. 1959;26:899-918

4 Adler RH. Konversion. In: Uexküll Th v. et al. Psychosomatische Medizin. 7. Aufl. München: Urban und Fischer; 2013, S. 691-9.

5 Schwarz AM, Adler RH, Schaechinger H, Goetz SM. Changes of high frequency heart rate variability during periods of intensive affects of helplessness/hopelessness in championship chess games. Psychosom Med. 2003;65:658-61.

6 Adler RH. Von der Biomedizin zur biopsychosozialen Medizin. Stuttgard: Schattauer; 2014, S. 56-66.

7 Adler RH. Hypothesenbildung bei Charles S. Peirce, Conon Doyle's Sherlock Holmes, Sigmund Freud und die moderne Neurobiologie. Schweiz Med Rundschau PRAXIS. 2006;95:767-71 